Conclusions DOTS awareness is still low among doctors who graduated before the introduction of DOTS. Private practitioners harboured myths and misconceptions about DOTS.

\section{P1-259 RELATIONS BETWEEN BMI AND TOTAL AND CAUSE SPECIFIC MORTALITY IN JAPAN: AGES COHORT}

\section{doi:10.1136/jech.2011.142976e.51}

${ }^{1} \mathrm{M}$ Nakade, ${ }^{*} \mathrm{~T}$ Ojima, ${ }^{3} \mathrm{H}$ Hirai, ${ }^{4} \mathrm{~J}$ Aida, ${ }^{5} \mathrm{~T}$ Hanibuchi, ${ }^{3} \mathrm{~K}$ Kondo. ${ }^{1}$ Tokaigakuen University, Nagoya, Japan; ${ }^{2}$ Hamamatsu University School of Hamamatsu, Japan; ${ }^{3}$ Nihon Fukushi University, Nagoya, Japan; ${ }^{4}$ University College London, London, UK; ${ }^{5}$ Ritsumeikan University, Kyoto, Japan

Purpose Within OECD countries, Japan has the lowest percentage $(3.4 \%)$ of the people with obesity ( $30 \leqq$ body mass index, BMI). This study aims to reveal the relationship between BMI and mortality among older Japanese.

Method We started a cohort study (Aichi Gerontological Evaluation Study, AGES) on non- institutionalised elderly aged 65 and over in 2003. We excluded the subjects with missing data and who died within 1 year in 4 year follow-up period considering reverse causation. Consequently, 17017 subjects among 21047 respondents for baseline survey were analysed. Cox's proportional hazard model was applied to calculate HR and 95\% CI of BMI for all cause and cause specific mortality. Age, disease history, weight loss, smoking, alcohol drinking, and exercise were used as covariates.

Result Respondents with lowest category of BMI (under 18.5) had the highest all cause mortality among both sexes. Compared to the respondents with BMI 23.0-24.9, men respondents with under 18.5 BMI and BMI 18.5-22.9 had significantly higher BMI (2.16 $(1.58-2.95, \mathrm{p}<0.001)$ and $1.30(1.04-1.63, \mathrm{p}=0.015)$ respectively). For cause specific mortality, men with BMI under 18.5 had a significantly higher $\mathrm{HR}$ of malignant neoplasm mortality $(\mathrm{HR}=1.68$ $(1.05-2.67, \mathrm{p}=0.030))$ and respiratory disease mortality $(\mathrm{HR}=5.63$ $(2.57-12.34, \mathrm{p}<0.001))$.

Conclusion Older Japanese with lower BMI had a significantly higher risk of all cause mortality in both sexes. Not only obesity, but also underweight prevention is needed for older Japanese.

\section{P1-260 DENTAL FLUOROSIS IN CHILDREN OF SÃO PAULO, BRAZIL, IN 1998-2008}

doi:10.1136/jech.2011.142976e.52

${ }^{1} \mathrm{P}$ C Narvai, ${ }^{3} \mathrm{M} C$ Soares, ${ }^{2} \mathrm{~A} C$ Frias, ${ }^{3} \mathrm{R}$ A A Marques, ${ }^{*}{ }^{3} \mathrm{D} S \mathrm{~S}$ Cruz, ${ }^{1} \mathrm{P}$ Frazao, 'J L F Antunes. 'School of Public Health, University of Sao Paulo, Sao Paulo, Brazil; ${ }^{2}$ School of Dentistry, University of Sao Paulo, Sao Paulo, Brazil; ${ }^{3}$ Health Department, Sao Paulo, Brazil

Introduction Water fluoridation and fluoride dentifrice contribute effectively in caries prevention. Both resources have nearly universal coverage in Sao Paulo, Brazil, since the 1980s. However, multiple exposures to fluoride demand the surveillance of dental fluorosis. This study aimed to assess fluorosis prevalence from 1998 to 2008. Methods Epidemiological surveys of oral health conducted in 1998 $(n=244), 2002(n=253)$ and in $2008(n=4249)$ informed fluorosis prevalence among 12-year-old school children. Although using different sample sizes, all surveys were considered representative for the city. Dental examinations were performed at schools, under natural illumination. The assessment of dental fluorosis used the index proposed by Dean in 1942, and recommended by the WHO for oral health surveys in 1997. Data analysis used the comparison of proportions included in the Epi-Info software.

Results Fluorosis prevalence was $43.9 \%$ (95\% CI $37.7 \%$ to $50.1 \%$ ) in $1998,33.2 \%$ (27.7\% to $39.2 \%$ ) in 2002 , and $39.1 \%$ (37.6\% to $40.6 \%$ ) in 2008. Mild and very mild fluorosis accounted for $88 \%$ of cases observed in 1998, $95 \%$ in 2002 , and $94 \%$ in 2008 . Less than $1 \%$ of examined children had severe fluorosis in 1998 and 2008; no cases were observed in 2002. No statistically significant difference was observed for the overall prevalence and for rates that specifically assessed differential levels of fluorosis during the period.

Conclusion The prevalence of dental fluorosis among children in Sao Paulo was stationary in the period 1998 to 2008. Most of the prevalence refers to mild and very mild levels of fluorosis, with no impact in function and aesthetics.

\section{P1-261 PREDICTION OF DROPOUT TUBERCULOSIS TREATMENT ON PRIORITARY CITIES TO CONTROL IN ESPÍRITO SANTO STATE, BRAZIL}

doi:10.1136/jech.2011.142976e.53

T N do Prado, ${ }^{*}$ R Dietze, A R Netto, E Zandonade, E L N Maciel. Universidade Federal do Espírito Santo, Vitoria, Espírito Santo, Brazil

Objectives To identify the epidemiological factors related to the abandonment of TB treatment in the priority municipalities for TB control and to establish a score for use in TB control programs to identify patients most likely to abandon treatment.

Methods case-control study matched by sex and notification place to compare patients who dropped out of the treatment (cases) with those who were cured (control) in eight priority municipalities for TB control in the Espirito Santo state, from January 2006 to July 2008. Patients were interviewed directly by one of the researchers, at the clinic or at home. To data analysis we performed a bivariate analysis and the significant results obtained from these analysis were for the logistic regression analysis and network neural artificial (NNA). The questionnaire score was created and validated.

Results The study involved 21 cases and 41 controls. In the bivariate analysis, the epidemiological factors involved in the TB treatment dropout were identified as follows: average income, smoking, age, occupation, religion, drugs, previous treatment for $\mathrm{TB}$ and willingness to abandon. The logistic regression analysis and neural network revealed that the use of illicit drugs and patient without religion was strongly related to the abandonment. It was found that the neural classification was not more effective than logistic regression for the score marks preparation.

Conclusions The score created was able to estimate the treatment dropout cases identified in the study and can be used in programs of $\mathrm{TB}$ control to identify patients most likely to abandon $\mathrm{TB}$ treatment.

\section{P1-262 POST-MORTEM INFECTION CONTROL IN JAPAN}

doi:10.1136/jech.2011.142976e.54

M Nishiyama,* H Sakuda, H Kawasaki, Y Shigematsu, R Doi, P D’Angelo, M Kakehashi. Hiroshima Bunkyo Women's University, Hiroshima, Japan

Introduction Healthcare workers in Japan lack the required level of understanding when dealing with cadavers at home and have the continuous and conflicting problem of balancing traditional, cultural beliefs with providing high quality infection control methods.

Objective The objectives of this research were to clarify the reasons for poor infection control post-mortem and to statistically show the significance of the problem.

Methods In 2006, 4773 participants gave feedback from a questionnaire. Participants were drawn from 13 different types of healthcare. They completed questionnaires about prevention and control of infection from cadavers. Questions covered four main areas: (1) The management of cadavers, (2) The role of nursing staff, 